\title{
Evidencias de Validez y Confiabilidad de las Puntuaciones del Cuestionario de Agresividad (AQ) para Adultos Argentinos
}

\author{
Validity and Reliability of the Aggression Questionnaire (AQ) for Argentine Adults
}

\author{
Zoilo Emilio García-Batista ${ }^{1}$, Luciana Sofía Moretti $^{2}$, Paul Franco $^{3}$, Leonardo Adrián Medrano $^{4}$ y \\ Alba Elisabeth Mustaca ${ }^{5}$
}

\begin{abstract}
Resumen
Según el Sistema Nacional de Información Criminal, en Argentina existe un crecimiento sostenido de delitos y problemas asociados a la agresividad. Además, se observa un aumento en la cantidad de mujeres que fueron víctimas de violencia durante el último año. A pesar de esta problemática, no se cuenta con instrumentos psicométricos debidamente adaptados a la población de adultos. Para validar el Cuestionario de Agresividad (AQ) se contó con la participación de 799 adultos (65,8\% eran mujeres). Estudios previos proponen estructuras factoriales que varían entre los dos y cuatro factores, por lo que se comparó el ajuste de los diferentes modelos. El modelo de tres factores (hostilidad, violencia verbal y violencia física) presentó un mejor ajuste. Los niveles de consistencia interna resultaron óptimos (valores $\alpha$ comprendidos entre .88 y .79). Asimismo, se obtuvieron evidencias favorables de validez al correlacionar las puntuaciones del AQ con medidas de Intolerancia a la Frustración.
\end{abstract}

Palabras clave: agresividad, validez, fiabilidad, análisis factorial confirmatorio

\begin{abstract}
According to the National Criminal Information System, in Argentina there is a sustained growth in crimes and problems associated with aggressiveness. In addition, there is an increase in the number of women who have been victims of violence in the last year. Despite this problem, there are no psychometric instruments properly adapted to the adult population. The validation of the Aggression Questionnaire (AQ) was done with 799 adult participants $(65.8 \%$ were women). Previous studies propose factorial structures that vary between two and four factors, so we compared the fitting of different models. The three-factor model (hostility, verbal violence, and physical violence) presented a better fit. Internal consistency levels were optimal ( $\alpha$ values between .88 and .79). Likewise, favorable evidence of validity was obtained by correlating AQ scores with measures of Frustration Intolerance.
\end{abstract}

Keywords: aggressiveness, validity, reliability, confirmatory factor analysis

\footnotetext{
${ }^{1}$ Psicólogo, PhD, Cognición Emoción y Estrés. Pontificia Universidad Católica Madre y Maestra -Escuela de Psicología, Santiago de los Caballeros, Republica Dominicana.

${ }^{2}$ Psicóloga, PhD en Psicología Clínica, Universidad Siglo 21, Córdoba, Argentina. Pontificia Universidad Católica Madre y Maestra, Santiago de los Caballeros, Republica Dominicana.

${ }^{3}$ Psicólogo, Lic. en Psicología, Universidad Abierta Interamericana- Facultad de Psicología y Humanidades. Docente de la Universidad Abierta Interamericana (UAI) e investigador del Centro de Altos Estudios en Ciencias Humanas y de la Salud (CAECIHS). Correo: rimbaud64@gmail.com

${ }^{4}$ Psicólogo, PhD en Psicología Clínica, Universidad Siglo 21, Córdoba, Argentina. Pontificia Universidad Católica Madre y Maestra, Santiago de los Caballeros, Republica Dominicana.

${ }^{5}$ Doctora en Psicología. Directora de proyectos de investigación del Consejo Nacional de Investigaciones Científicas y Tecnológicas (CONICET), Secretaría de Ciencia y Tecnología (SECYT) y de la Universidad Abierta Interamericana (UAI). Correo: albamustaca@gmail.com; alba.mustaca@uai.edu.ar (Autor de correspondencia)

Revista Iberoamericana de Diagnóstico y Evaluación - e Avaliação Psicológica. RIDEP · N62 · Vol.1 · 17-28 · 2022

ISSN: 1135-3848 print /2183-6051online
} 


\section{Introducción}

Aunque la agresividad es inherente al comportamiento humano, constituye un factor de riesgo para diferentes formas de conducta antisocial, como la delincuencia o la violencia de pareja (Pechorro et al., 2015; Redondo et al., 2017). Debido a las enormes consecuencias que tienen las conductas agresivas, la Organización Mundial de la Salud declaró a los problemas asociados a la agresividad y la violencia como uno de los principales problemas de salud pública en todo el mundo (OMS, 2003).

Probablemente la definición más aceptada de agresividad sea la propuesta por Buss (1961), quien afirma que se trata de una respuesta que un organismo realiza con el objetivo de dañar a otro organismo. Sin embargo, su definición continúa siendo objeto de controversia debido a la complejidad del constructo, la variedad de formas que puede adoptar $\mathrm{y}$ al hecho de estar estrechamente emparentado a otros constructos tales como violencia, hostilidad e ira (Contini, 2015).

Aunque la violencia se trata a veces como un constructo separado de la agresión, existe consenso en considerarla como un subtipo de agresión. De esta manera, la violencia refiere a una forma extrema de agresión. La mejor manera de conceptualizar los comportamientos agresivos $\mathrm{y}$ violentos es situarlos en un continuo de gravedad, con actos de agresión relativamente menores (por ejemplo, empujones) en el extremo inferior del espectro y violencia (por ejemplo, homicidio) en el extremo superior. Así, todos los actos de violencia se consideran casos de agresión, pero no todos los actos de agresión se consideran casos de violencia (Allen \& Anderson, 2017).

Asimismo, cabe realizar algunas precisiones conceptuales. Mientras agresión se refiere a un comportamiento específico, la agresividad hace referencia a una disposición a actuar de manera agresiva. Por su parte, la ira constituye un estado emocional, mientras la hostilidad hace referencia a las actitudes que acompañan los comportamientos agresivos. De esta manera, la hostilidad representaría un componente más cognitivo, mientras que la ira constituiría un componente emocional de la agresividad (Contini, 2015).
La agresión puede adoptar muchas formas, desde actos relativamente menores (como insultos o empujones) hasta actos más graves (como golpes, patadas o puñetazos) o actos severos (como apuñalar, disparar o matar). El hecho de que la agresión aparezca de tantas formas puede dificultar a veces la determinación de si se ha producido o no una agresión (Allen \& Anderson, 2017). Esta característica ha afectado notablemente la forma de medir el constructo.

La multiplicidad de formas que puede adoptar la agresión trae aparejado diversos cuestionarios y escalas que permitan abordar cada una de dichas manifestaciones. Por ejemplo para el fenómeno de la violencia en la pareja (López-Cepero Borrego et al., 2015); para la violencia social encubierta hacia las mujeres (Vinagre-González et al., 2020); para la valoración de la conducta violenta y el riesgo de reincidencia (Jiménez-García et al., 2010), para la violencia filio-parental (JiménezGarcía et al., 2019), entre otras.

El Cuestionario de Agresión (Aggression Questionnaire; AQ) propuesto por Buss y Perry (AQ, 1992), se convirtió en el "gold standard" en el estudio de la conducta agresiva (Gallardo-Pujol et al., 2019). Fue creado a partir del intento de modernizar el inventario de hostilidad (Buss Durkee Hostility Inventory; BDHI; Buss \& Durkee, 1957). El BDHI estaba formado por 75 ítems y tenía una estructura bifactorial formada por un componente actitudinal de la agresión (negativismo, resentimiento y sospecha) y otro componente 'motor' (asalto, agresión indirecta, irritabilidad y agresión verbal), con un formato de respuesta dicotómico (verdadero, falso). Diferentes estudios dejaron expuestas ciertas limitaciones del instrumento, sobre todo en lo referido a su estructura factorial (Redondo et al., 2017; Vigil-Colet et al., 2005). Por otra parte, el formato de respuesta verdadero-falso no era el más apropiado (Valdivia-Peralta et al., 2014). Es por ello que Buss y Perry (1992) utilizaron algunos de los ítems originales compuestos por Buss y Durkee, reescribieron otros, eliminaron aquellos que resultaban ambiguos y añadieron nuevos, creando el cuestionario definitivo compuesto por 29 afirmaciones (Buss \& Perry, 1992).

La escala AQ ha sido traducida y utilizada en múltiples países debido a su fácil utilización y su 
bajo costo. Su estructura factorial ha sido estudiada en idiomas y culturas como la japonesa (Nakano, 2001; Ramírez et al., 2001), eslovaca (Lovas \& Trenkova, 1996), rusa (Ruchkin \& Eisemann, 2000), alemana (Von Collani \& Werner, 2005), holandesa (Meesters et al., 1996), francesa (Pfister \& Masse, 2001) y italiana (Fossati et al., 2003; Sommantico et al., 2008). La estructura de la escala ha demostrado ser parcialmente invariante con respecto al idioma, cultura, género y edad (Ang, 2007; Bryant \& Smith, 2001; Fossati et al., 2003; Reyna et al., 2011; Santisteban et al., 2007; Vitoratou et al., 2009).

En idioma español la estructura factorial ha sido evaluada en España por Andreu, Peña y Graña (2002) quienes, utilizando una muestra de 1382 jóvenes, obtuvieron factores consistentes: para la agresión física $(\alpha=.86)$, ira $(\alpha=.77)$, agresión verbal $(\alpha=.68)$ y hostilidad $(\alpha=.72)$. Los estudios posteriores reportaron resultados semejantes (García-León et al., 2002; Porras et al., 2002). La adaptación del instrumento en preadolescentes y adolescentes confirmaron la misma estructura (Santisteban et. al, 2007). Asimismo, se han evaluado las propiedades psicométricas a una muestra de 767 maltratadores en tratamiento psicológico, en España. Los resultados del análisis factorial confirmatorio apoyaron la estructura de cuatro factores, concluyendo que es un instrumento adecuado para evaluar agresión, ira y hostilidad en hombres que maltratan a sus parejas (Redondo et al., 2017). Además, el AQ ha mostrado validez de criterio para distinguir entre reclusos carcelarios y estudiantes (García-León et al., 2002).

Aunque la mayoría de los estudios apoyan una estructura de cuatro factores, hay discrepancias con respecto a los ítems asignados a cada factor (Andreu et al., 2020; García-León et al., 2002). Además, algunos investigadores han encontrado mejores ajustes con dos factores (Maxwell, 2007; Williams et al.,1996) y tres factores (Sommantico et al., 2008).

En relación con el género, muchos estudios muestran diferencias en el factor agresión física, ya que los hombres puntúan sistemáticamente más que las mujeres (por ej. Andreu et al., 2002; Buss \& Perry, 1992; Tremblay \& Ewart, 2005; para una revisión ver Archer, 2004). En cambio, en referencia a la agresión verbal, la evidencia es menos clara mostrando un patrón mixto y efectos de tamaño de la muestra pequeño (Ang, 2007; Buss \& Perry, 1992; García-León et al., 2002; Meesters et al., 1996; Sommantico et al., 2008; Vitoratou et al., 2009). Respecto a la ira, no pocas veces aparece una diferencia, al ser el puntaje más alto en las mujeres que en los hombres (por ej. Andreu et al., 2002; Santisteban et al., 2007; Sommantico et al., 2008; Von Collani \& Werner, 2005). Finalmente, en hostilidad rara vez se han informado resultados con pocas y variadas diferencias (por ej., Andreu et al., 2002; Buss \& Perry, 1992; Maxwell, 2007).

En América Latina la escala AQ se validó en Colombia, el Salvador, México, Perú, Chile y Argentina. En Colombia, se partió de la versión original del $\mathrm{AQ}$, a la que le agregaron 11 ítems (Castrillón et al., 2004). En este estudio se observó una estructura de cinco factores añadiendo a los originales el factor de No Agresión. Las validaciones realizadas en El Salvador (Sierra \& Gutiérrez, 2007), México (Cruz, 2013), Perú (Matalinares Calvet et al., 2012) y Chile (Valdivia-Peralta et al., 2014), obtuvieron evidencias favorables para una estructura subyacente de cuatro factores y niveles aceptables de consistencia interna.

En Argentina, Reyna et al. (2011) llevaron adelante una adaptación de la versión de Andreu et al. (2002) en una muestra de 371 adolescentes cordobeses de 12 a 19 años $(\mathrm{M}=14,89 ; \mathrm{SD}=1,97)$, encontrando dos factores en un primer estudio exploratorio y luego una estructura de 2 y 4 factores en el análisis confirmatorio.

Debido a que la escala AQ fue validada en Argentina solamente en una población adolescente, el presente trabajo tiene por objetivo principal recabar evidencia de validez para las puntuaciones del AQ en una población adulta de Argentina. Tomando en consideración que estudios previos proponen estructuras factoriales que varían entre los dos y cuatro factores, en el presente trabajo se comparará el ajuste de los diferentes modelos mediante análisis factorial confirmatorio. Por otra parte, se analizará la consistencia interna de cada escala y se recabará evidencia de validez test-criterio correlacionando las puntuaciones del AQ con las puntuaciones de la Escala de Intolerancia a la Frustración, ya que 
se trata de una variable estrechamente vinculada con la agresividad (Medrano et al., 2018). Por último, considerando que existen abundantes estudios antecedentes que sugieren diferencias en los niveles de agresividad según el género, se contrastarán puntajes entre dichos grupos y realizarán estudios de invarianza.

\section{Método}

\section{Participantes}

La muestra estuvo formada por 799 argentinos (edad media=29.36 años; $\mathrm{DT}=11.61$ ) seleccionados a partir de un muestreo no probabilístico accidental. El $65.8 \%$ eran mujeres, el $33.2 \%$ hombres y un $1 \%$ se identificó en la categoría de "Otros". En cuanto al nivel de educación la mayoría contaba con estudios superiores dado que el $33.2 \%$ estaba en cursos terciarios o universitarios incompleto, el $23.2 \%$ universitario completo y un $5.8 \%$ estudios de posgrado; el $20.7 \%$ contaba con secundario incompleto, un $16.6 \%$ con secundario completo y un $0.3 \%$ alcanzó solamente la primaria completa.

\section{Instrumentos}

Cuestionario de Agresión (AQ, Buss \& Perry, 1992), versión en castellano validada por Andreu Rodríguez et al. (2002). Es autoadministrado, cuenta con 29 ítems respondidos en una escala tipo Likert de cinco puntos (desde 1="Es nada característico de mí" a 5="Es muy característico de mí"). Esta versión presenta cuatro factores subyacentes: agresividad física $(\alpha=86)$, agresividad verbal $(\alpha=.68)$, ira $(\alpha=.77)$ y hostilidad $(\alpha=.72)$. Las propiedades psicométricas de las puntuaciones de este instrumento en población argentina serán recabadas en el presente trabajo.

Escala de Intolerancia a la Frustración (Frustration Discomfort Scale, EIF; Harrington, 2005). Se trabajó con la versión adaptada por Medrano et. al. 2018). Cuenta con 21 ítems que los sujetos deben responder utilizando una escala Likert de 5 puntos que van desde $1=$ "No es nada característico de mí" hasta 5="Es muy característico de mí". A mayor puntuación mayor intolerancia a la frustración o bien menor tolerancia a la misma. La EIF cuenta con una estructura de 4 factores: "Intolerancia a la incomodidad" $(\alpha=.61)$, "Derechos" $(\alpha=.76)$, "Intolerancia emocional" $(\alpha=.76)$ y "Logro" $(\alpha=.55)$. Las puntuaciones de la escala total son los únicos que presentan valores óptimos $(\alpha=.84)$; mientras que las correlaciones ítem-total corregidas presentaron valores entre .34 y .59 .

\section{Procedimiento}

La aplicación de los cuestionarios se realizó en lugares públicos y organizaciones que accedieron a cooperar con el estudio. También se convocó a participantes a través de redes sociales. $\mathrm{La}$ participación en la investigación fue voluntaria y antes de responder el cuestionario los participantes dieron su consentimiento informado. Del total de participantes, el $45.7 \% \quad(n=365)$ respondió los instrumentos on line a través de la plataforma Google Form y el 54.3\% ( $\mathrm{n}=434)$ lo hizo de manera presencial, en papel. No se observaron diferencias estadísticamente significativas entre ambas muestras en cuanto a la distribución del sexo $\left(\chi^{2}=.55 ; \mathrm{gl}=1 ; p=.46\right)$ y la edad $(\mathrm{t}=.76 ; \mathrm{gl}=764$; $p=.44)$ de los participantes.

\section{Análisis de datos}

Los datos obtenidos fueron cargados en el programa SPSS versión 20 (George \& Mallery, 2010). A continuación, se realizó un análisis de exploración inicial de datos para evaluar la presencia de casos atípicos, valores perdidos y examinar el supuesto de normalidad univariada y multivariada. Luego, se efectuaron estudios para evaluar la estructura interna mediante el uso del análisis factorial confirmatorio (AFC) a través del programa AMOS 20 (Arbuckle, 2012). Tal como sugiere la literatura se utilizaron múltiples indicadores de ajuste (Bentler, 2007; Byrne, 2001). Más concretamente, el estadístico chicuadrado, el índice de ajuste comparativo (CFI), el índice de bondad de ajuste (GFI), y el error cuadrático medio de aproximación (RMSEA). Se utilizaron los puntos de corte reportados por $\mathrm{Hu}$ y Bentler (1999), quienes recomiendan valores superiores a .95 para los índices CFI y GFI para considerar un ajuste óptimo, y superiores a .90 para un ajuste aceptable. Por otra parte, valores inferiores a .06 para el RMSEA se consideran óptimos e inferiores a .08 aceptables. 
Tabla 1. Índices de ajuste modelos AQ

\begin{tabular}{lccccccc}
\hline & $\chi^{2}$ & $\mathrm{gl}$ & $\chi^{2} / \mathrm{gl}$ & CFI & GFI & TLI & RMSEA \\
\hline Modelo Dos factores & $2115.22^{*}$ & 323 & 4.73 & .74 & .80 & .72 & .08 \\
Modelo Tres Factores & $772.785^{*}$ & 207 & 4.79 & .90 & .92 & .90 & .06 \\
Modelo Cuatro factores & $2928.50^{*}$ & 205 & 3.88 & .74 & .84 & .73 & .10 \\
\hline
\end{tabular}

Nota. $* p<.01$

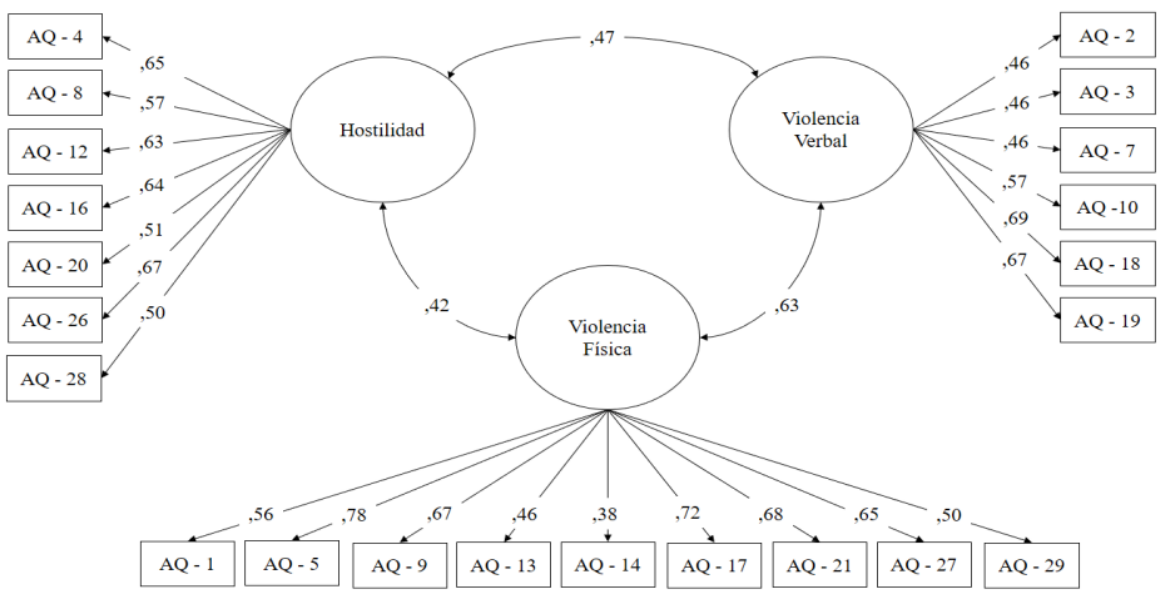

Figura 1. Análisis Factorial Confirmatorio del AQ

Se evaluó la consistencia interna utilizando el estadístico $\alpha$ de Cronbach, y se realizaron estudios tendientes a aportar evidencias de validez testcriterio correlacionando mediante el coeficiente $r$ de Pearson las puntuaciones de AQ con las puntuaciones de la "Escala de Intolerancia a la Frustración". Asimismo, y para analizar con mayor profundidad la relación entre las dimensiones de ambos instrumentos, se efectuó un análisis de regresión múltiple.

Con el objetivo de analizar si el modelo se mantenía invariante en función del sexo de los participantes se realizó un análisis multigrupo con ecuaciones estructurales. Para ello se atendió al cambio en el coeficiente $\chi^{2}$, y se utilizó como criterio adicional que el cambio en el coeficiente CFI no fuese superior a .01 (Cheung \& Rensvold, 2002).

\section{Resultados}

\section{Validez de la Estructura Interna}

En primera instancia, se examinó el cumplimiento de los supuestos estadísticos y se analizó el patrón de casos perdidos y valores atípicos. Ningún ítem presentó más del 5\% de valores perdidos, por lo que se optó por utilizar el método de eliminación por caso ("listwise") para el tratamiento de dichos valores (Coenders et al., 2005). Para evaluar la presencia de casos atípicos univariados se calcularon las puntuaciones $\mathrm{z}$ para cada ítem, considerando como valores atípicos aquellos que presentaran valores fuera del rango $\mathrm{z}$ \pm 3 (George \& Mallery, 2010). Para identificar casos atípicos multivariados se utilizó el procedimiento estadístico de la distancia de Mahalanobis ( $\left.\mathrm{D}^{2}\right)$. No se observaron casos atípicos univariados, y la cantidad de casos atípicos multivariado fue inferior al $5 \%$, no afectando normalidad multivariada de la distribución (Mardia=47,33). Cabe señalar además que todos los ítems presentaron índices de asimetría y curtosis comprendidos entre \pm 2 , observándose un leve alejamiento de la normalidad (George \& Mallery, 2010).

Para realizar los estudios de AFC se siguieron los pasos recomendados por la literatura (Medrano \& Muñoz, 2017). En primer lugar, se especificaron tres modelos: modelo de dos factores, tres factores y cuatro factores. Se procedió a la estimación de los modelos utilizando el método de estimación por Máxima-Probabilidad, ya que es el método más recomendado cuando los ítems presentan una distribución normal (Iacobucci, 2010). Ninguno de los modelos presentó un ajuste aceptable, por lo cual se exploró la saturación factorial de los reactivos. Siguiendo las recomendaciones de George y Mallery (2010), se eliminaron reactivos que no presentaban saturaciones superiores a $.40 \mathrm{en}$ 
Tabla 2. Evidencias de validez test-criterio entre las puntuaciones del AQ y EIF

\begin{tabular}{lccc}
\hline & Agresividad Física & Agresividad Verbal & Hostilidad \\
\hline Intolerancia a la Incomodidad & $.12 * *$ & $.15 * *$ & $.32 * *$ \\
Derecho & $.30 * *$ & $.45^{* *}$ & $.45^{* *}$ \\
Intolerancia Emocional & $.08 *$ & $.20^{* *}$ & $.32 * *$ \\
Logro & .06 & $.19 * *$ & $.18^{* *}$ \\
Escala Total & $.21 * *$ & $.37 * *$ & $.46^{* *}$ \\
\hline Nota. $* p<.05 ; * * p<.01$ & & &
\end{tabular}

alguno de los factores. En función de ello se eliminaron los ítems $6,11,22,23,24,15$, y 25. A continuación, se evaluó el ajuste de cada modelo considerando múltiples índices de ajuste, los resultados obtenidos se presentan en la Tabla 1. Tal como puede apreciarse solo el modelo de tres factores presentó un ajuste aceptable.

\section{Consistencia Interna}

Se evaluó la consistencia interna de cada factor y la escala general mediante el coeficiente $\alpha$ de Cronbach. Asimismo, se calculó la correlación ítem-total corregida para los ítems de cada factor. Los resultados obtenidos fueron: $\alpha=.84$ para violencia física (correlaciones ítem-total corregidas entre .49 y .72); $\alpha=.80$ para violencia verbal (correlaciones ítem-total corregidas entre .45 y .61 ); y $\alpha=.79$ para hostilidad (correlaciones ítem-total corregidas entre .46 y .61). Por su parte, la escala total fue la única que presentó valores óptimos $(\alpha=.88 ; \quad$ correlaciones ítem-total corregidas entre .36 y .55$)$.

\section{Validez de Criterio}

Con el objeto de aportar evidencias sobre la validez test-criterio, se correlacionaron las puntuaciones de AQ con las puntuaciones de la EIF. Tal como se esperaba se obtuvieron correlaciones directas y significativas en todas las escalas (Tabla 2).

Con el objetivo de aportar mayores evidencias de validez, se realizaron análisis de regresión múltiple entre las dimensiones del EIF y las puntuaciones del AQ (Tabla 3). Los resultados obtenidos indican que la dimensión de Derecho de la EIF se asocia significativamente con todas las dimensiones del AQ. Se observa una diferencia respecto a la dimensión de Hostilidad, donde también se aprecia una relación significativa con la intolerancia emocional y a la incomodidad.

\section{Análisis de Invarianza según sexo}

Para evaluar si el modelo se mantenía invariante en función del sexo de los participantes se procedió a realizar un análisis de invarianza. Siguiendo las recomendaciones de Brown et al. (2014) se verificó inicialmente la invarianza configural (equivalencia en la configuración del modelo de medida), y posteriormente se introdujeron restricciones progresivas para examinar la invarianza métrica (equivalencia en las saturaciones factoriales), fuerte (equivalencia en los valores interceptuales) y estricta (equivalencia entre las varianzas de error). En cada caso la invarianza puede ser asumida si no se observan diferencias significativas entre el ajuste del modelo de parámetros libres y el ajuste del modelo con parámetros restringidos.

En relación con el sexo se verificó la invarianza configural $\left(\chi^{2}=1014 ; \mathrm{gl}=402 ; \mathrm{CFI}=.90\right)$, métrica $\quad\left(\Delta \chi^{2}=35.403 ; \quad \Delta \mathrm{gl}=19 ; \quad p=.12\right.$; $\Delta \mathrm{CFI}=0.002)$, fuerte $\left(\Delta \chi^{2}=48.65 ; \Delta \mathrm{gl}=26 ; p=.05\right.$; $\Delta \mathrm{CFI}=0.003)$ y estricta $\left(\Delta \chi^{2}=190.88 ; \Delta \mathrm{gl}=52\right.$; $p=.01 ; \Delta \mathrm{CFI}=0.003)$. Cabe destacar que, aunque el cambio en el coeficiente $\chi^{2}$ fue significativo, en ningún caso se observó un cambio en el índice CFI mayor a .01. Este último criterio es el más recomendado atendiendo a la sensibilidad de $\chi^{2}$ cuando se trabajan con muestras de gran tamaño (Barrera-Barrera et al., 2015).

\section{Diferencias de contraste de grupos según sexo y formato de administración}

Se llevaron a cabo prueba $t$ de Student para determinar si existían diferencias en las puntuaciones del AQ según el sexo de los participantes. Al evaluar el supuesto de homogeneidad de la varianza mediante el contraste de Levene no se obtuvieron valores significativos. Los resultados obtenidos indican que solo existe una diferencia significativa entre hombres y mujeres en la dimensión de agresividad física ( $t=6.98 ; \mathrm{gl}=789 ; p<.01)$. De esta forma, se puede apreciar que los hombres (Media $=20.35$; 
Tabla 3. Análisis de regresión múltiple, evidencias de validez test-criterio entre las puntuaciones del AQ y EIF

\begin{tabular}{|c|c|c|c|c|c|c|c|c|c|c|c|c|}
\hline \multirow{2}{*}{$\begin{array}{l}\text { Variable } \\
\text { dependiente }\end{array}$} & \multirow{2}{*}{ Variable Independiente } & \multirow{2}{*}{$r^{2}$} & \multirow{2}{*}{$\mathrm{r}^{2} \mathrm{~A}$} & \multirow{2}{*}{ RMSE } & \multirow{2}{*}{$\mathrm{F}$} & \multirow{2}{*}{ B } & \multirow{2}{*}{$\mathrm{DE}$} & \multicolumn{2}{|c|}{$95 \% \mathrm{IC}$} & \multirow{2}{*}{$\beta$} & \multirow{2}{*}{$\mathrm{T}$} & \multirow{2}{*}{$p$} \\
\hline & & & & & & & & Inferior & Superior & & & \\
\hline AQ Total & & .26 & .26 & 13.67 & 7.91 & & & & & & & .000 \\
\hline \multirow{9}{*}{ Hostilidad } & Intolerancia Emocional & & & & & .06 & .11 & -.16 & .29 & .02 & .56 & .57 \\
\hline & Logro & & & & & -.21 & .19 & -.58 & .17 & -.04 & -1.09 & .28 \\
\hline & Derechos & & & & & 1.47 & .11 & 1.25 & 1.69 & .50 & 13.30 & .00 \\
\hline & Intolerancia Incomodidad & & & & & .21 & .18 & -.15 & .58 & .04 & 1.15 & .25 \\
\hline & & .23 & .23 & 5.56 & 6.40 & & & & & & & .000 \\
\hline & Intolerancia Emocional & & & & & .16 & .05 & .07 & .25 & .13 & 3.47 & .00 \\
\hline & Logro & & & & & -.08 & .08 & -.23 & .07 & -.04 & -1.02 & .31 \\
\hline & Derechos & & & & & .40 & .04 & .31 & .49 & .34 & 8.84 & .00 \\
\hline & Intolerancia Incomodidad & & & & & .32 & .08 & .17 & .47 & .15 & 4.29 & .00 \\
\hline \multirow[t]{5}{*}{$\begin{array}{l}\text { Violencia } \\
\text { verbal }\end{array}$} & & .20 & .20 & 5.00 & 51.30 & & & & & & & .000 \\
\hline & Intolerancia Emocional & & & & & .00 & .04 & -.09 & .08 & .00 & -.08 & .94 \\
\hline & Logro & & & & & .01 & .07 & -.13 & .15 & .01 & .15 & .88 \\
\hline & Derechos & & & & & .49 & .04 & .41 & .57 & .47 & 12.04 & .00 \\
\hline & Intolerancia Incomodidad & & & & & -.09 & .07 & -.23 & .04 & -.05 & -1.37 & .17 \\
\hline \multirow[t]{5}{*}{$\begin{array}{l}\text { Violencia } \\
\text { física }\end{array}$} & & .13 & .13 & 7.28 & 3.33 & & & & & & & .000 \\
\hline & Intolerancia Emocional & & & & & -.09 & .06 & -.21 & .03 & -.06 & -1.54 & .13 \\
\hline & Logro & & & & & -.14 & .10 & -.34 & .06 & -.05 & -1.36 & .17 \\
\hline & Derechos & & & & & .59 & .06 & .47 & .70 & .41 & 9.95 & .00 \\
\hline & Intolerancia Incomodidad & & & & & -.02 & .10 & -.21 & .18 & -.01 & -.17 & .87 \\
\hline
\end{tabular}

Desviación Estándar=8.22) presentan valores más elevados de violencia física que las mujeres (Media=16.36; Desviación Estándar=7.17). Al calcular el tamaño del efecto de la diferencia se obtiene un valor $d$ de Cohen de .52, lo que indica que se trata de una diferencia grande. No se observaron diferencias significativas en las restantes dimensiones del AQ.

Asimismo, se analizó si existían diferencias según el formato de administración (versión on line vs. lápiz y papel). En primera instancia se corroboró el supuesto de homogeneidad de la varianza al no obtener resultados significativos mediante el contraste de Levene. Los resultados obtenidos de la prueba $t$ de Student indican que no existen diferencias estadísticamente significativas en función del formato de administración al considerar el puntaje total AQ $(t=0.15 ; \mathrm{gl}=789$; $p=.88)$, y las dimensiones de hostilidad $(t=1.53$; $\mathrm{gl}=789 ; p=.12)$, violencia verbal $(t=1.59 ; \mathrm{gl}=789$; $p=.11)$ y violencia física $(t=0.42 ; \mathrm{gl}=789 ; p=.67)$.

\section{Discusión}

De acuerdo con Buss (1961), la agresividad se puede definir como la respuesta que efectúa un ser vivo para dañar a otro. Por ende, a pesar de ser una parte de la naturaleza humana, es posible que se convierta en un factor de riesgo para el desarrollo de conductas antisociales sumamente perjudiciales, como la delincuencia y la violencia de pareja (Pechorro et al., 2015; Redondo et al.,
2017). En consecuencia, evaluarla de forma correcta y precoz es esencial para un abordaje adecuado a nivel terapéutico, pudiendo así prevenir muchos problemas y consecuencias graves.

Una de las pruebas psicológicas más utilizadas para evaluar los distintos niveles de agresividad de una persona es el AQ propuesto por Buss y Perry (1992). Este se convirtió en el "gold standard" en el estudio de la conducta agresiva (Gallardo-Pujol et al., 2019). Sin embargo, este instrumento no contaba con estudios que examinen sus propiedades psicométricas en la población adulta de Argentina. La ausencia de instrumentos que evalúen de manera válida y confiable la agresividad limita seriamente las posibilidades de desarrollar investigaciones científicas que permitan la identificación de factores de riesgo o la evaluación de protocolos de intervención. Más aún, debemos considerar que según el Sistema Nacional de Información Criminal de Argentina (SNIC), se observa un crecimiento sostenido en delitos asociados a comportamientos violentos, tales como homicidios o lesiones. Asimismo, durante el año 2019 se reportó un crecimiento en la prevalencia de mujeres víctimas de violencia durante el último año (de un $7.3 \%$ a un $8.5 \%$ ). Por estas razones, la presente investigación se planteó como objetivo principal recabar evidencias de validez y confiabilidad de las puntuaciones del AQ para adultos argentinos. 
Debido a que la cantidad de factores reportados en investigaciones previas varía entre dos y cuatro factores (Andreu et al., 2002; García-León et al., 2002; Maxwell, 2007; Porras et al., 2002; Santisteban et al., 2007; Sommantico et al., 2008; Williams et al., 1996), se optó por realizar estudios de AFC para determinar la estructura subyacente más adecuada. Los resultados obtenidos indican que el modelo de tres factores (hostilidad, violencia verbal y violencia física) presenta un mejor ajuste, a diferencia de la versión original, donde se plantea un instrumento de cuatro factores (ira, hostilidad, violencia verbal $\mathrm{y}$ violencia física). Al analizar los motivos por lo cual se realiza una reducción de cuatro a tres factores es porque se observa que los ítems de los factores de ira y hostilidad tienden a agruparse en un mismo factor. Estos resultados indicarían un solapamiento entre ambos factores. Este hallazgo tiene implicancias teóricas y prácticas, dado que, aunque algunos autores utilizan el concepto de ira para referirse a una emoción, y el de hostilidad para denotar una actitud que motiva acciones agresivas (Fernández-Abascal \& Martín Díaz, 1994), los hallazgos empíricos indican que dicha diferenciación teórica es difícil de observar a nivel empírico. De hecho, son muchos los estudios que indican que las actitudes poseen un fuerte componente emocional, o bien, que existe una relación recíproca entre las actitudes y las emociones (Featherstone \& Zhang, 2020). Esto no equivale a afirmar que no existe una diferenciación entre ambos constructos; más bien se plantea la dificultad para redactar reactivos puros que permitan diferenciar a nivel empírico ambos constructos. De hecho, los ítems orientados a evaluar actitudes generalmente involucran componentes emocionales, generando un solapamiento entre ambos constructos.

En torno a la confiabilidad del AQ, se ratificó que la prueba completa tiene una consistencia interna óptima $(\alpha=.88)$; además, las subescalas evidenciaron una consistencia interna aceptable (valores $\alpha$ comprendidos entre .79 y .84). Esto va en consonancia con los resultados de otros estudios internacionales (López et al., 2009; Sierra \& Gutiérrez, 2007; Sommantico et al., 2008).

Los estudios tendientes a recabar evidencias de validez con fuentes externas indicaron que todas las subescalas presentaron correlaciones directas y significativas con la EIF. Estos resultados son coherentes con lo esperado en términos teóricos, ya que son diversos los estudios que denotan una relación entre las Intolerancia a la Frustración y la Agresividad (Medrano et al., 2018). Los resultados indican que las personas que presentan creencias referidas a que las otras personas no deberían interponerse en la consecución de los deseos personales, presentan mayores niveles de hostilidad, agresividad física y verbal. Estos resultados son coherentes con la literatura, donde se observa que la frustración por no poder complacer deseos, que son vistos como derechos por la propia persona, se asocia a una respuesta de hostilidad y violencia (Contini, 2015). Por otra parte, las creencias de intolerancia emocional (creencia de que no deberíamos experimentar emociones displacenteras) e intolerancia a la incomodidad (creencias de que la vida debería ser fácil, cómoda y libre de problemas), también se relacionan positivamente con los niveles de hostilidad, y no con las dimensiones de violencia del AQ. Estos resultados contribuyen a diferenciar entre hostilidad y violencia (Allen \& Anderson, 2017). Tal como puede apreciarse en los resultados de la regresión múltiple, la dificultad para lidiar con emociones displacenteras e incómodas puede generar respuestas de hostilidad, pero no se relaciona con comportamientos violentos verbales o no verbales.

Por último, es importante resaltar los datos analizados en este estudio que están relacionados con el sexo de los participantes. Por un lado, se evaluó la invarianza del modelo según el sexo. En la presente investigación se observó que el modelo de AQ se mantuvo invariante en torno al sexo. Por otro lado, se analizó si había diferencias en cuanto a los tipos de agresividad manifestados por los hombres y las mujeres. Se encontró que la agresividad física era significativamente mayor en hombres, coincidiendo este resultado con el de múltiples estudios antecedentes (Andreu et al., 2002; Buss \& Perry, 1992; Tremblay \& Ewart, 2005; Sierra \& Gutiérrez, 2007). En torno a los otros factores, no se hallaron diferencias significativas según el sexo.

Los resultados obtenidos deben analizarse contemplando una serie de limitaciones. En primera instancia, existen algunos factores que limitan la representatividad de la muestra 
analizada. La misma está compuesta por personas jóvenes (edad promedio de 29,36), fundamentalmente mujeres, y con un porcentaje alto de personas con estudios secundarios $\mathrm{o}$ universitarios. Un segundo aspecto a contemplar refiere a la necesidad de desarrollar estudios psicométricos adicionales, como por ejemplo la estabilidad temporal de las puntuaciones.

A pesar de las limitaciones señaladas, los resultados obtenidos en el presente trabajo sugieren que las puntuaciones del AQ constituyen una medida válida y confiable para el contexto argentino. Se cuenta así con un instrumento útil para el diagnóstico y evaluación de problemas asociados a la violencia y la agresión. No obstante, se requiere de nuevas investigaciones. A partir de las recomendaciones de las OMS (2003), se identifican tres líneas futuras de investigación. En primer lugar, desarrollar y adaptar nuevos instrumentos para aumentar la capacidad de recolectar datos sobre violencia. Aunque el AQ es un instrumento de amplio uso, sería provechoso contar con instrumentos adicionales a los fines de evaluar de manera amplia y exhaustiva variables asociadas a la agresividad. En segundo lugar, se deben diseñar y evaluar programas de intervención que permitan implementar respuestas de prevención primaria de la violencia. Al respecto cabe señalar que las intervenciones basadas en la evidencia constituyen un campo aún pobremente desarrollado en el contexto latinoamericano (Medrano \& Moretti, 2015). Finalmente, se requiere de investigaciones que busquen integrar la identificación temprana de perfiles agresivos con políticas sociales y educativas que posibiliten una prevención temprana de problemas asociados a la violencia.

Acorde con el informe mundial sobre violencia y salud de la OMS (2003), la violencia y la agresividad no constituyen un problema social sin solución o un componente ineludible de la condición humana. Se requiere de conocimientos, cooperación, innovación y compromiso para prevenir la violencia en todo el mundo. El presente trabajo pretende ser una contribución al respecto.

\section{Agradecimientos}

Esta investigación fue parcialmente financiada por Universidad Abierta Interamericana- Facultad de Psicología y Humanidades.

\section{Referencias}

Allen, J. J., \& Anderson, C. A. (2017). Aggression and violence: Definitions and distinctions. The Wiley handbook of violence and aggression, 1-14.

https://doi.org/:10.1002/9781119057574.whb $\mathrm{va} 001$

Andreu, J. M., Peña, M. E., \& Graña, J. L. (2002). Adaptación psicométrica de la versión española del Cuestionario de Agresión. Psicothema, 14, 476-482. Recuperado de http://www.redalyc.org/articulo.oa?id=727142 45

Ang, R. P. (2007). Factor structure of the 12 -item aggression questionnaire: Further evidence from Asian adolescent samples. Journal of Adolescence, 30, 671-685.

https://doi.org/:10.1016/j.adolescence.2006.05 .003

Arbuckle, J. L. (2012). IBM SPSS Amos 21. User's guide. IBM.

Archer, J. (2004). Sex differences in real-word settings: A meta-analytic review. Review of General Psychology, 8, 291-332. https://doi.org/:10.1037/1089-2680.8.4.291

Barrera-Barrera, R., Navarro-García, A., \& PerisOrtiz, M. (2015). El papel de la invarianza factorial en la validación del constructo calidad de servicio electrónico. Revista Europea de Dirección y Economía de la Empresa, 24(1), 13-24. https://doi.org/:10.1016/j.redee.2014.07.001

Bentler, P. (2007). On test and indices for evaluating structural models. Journal of Personality and Individuals Differences, 42, 825-829.

https://doi.org/:10.1016/j.paid.2006.09.024

Brown, S. D., Unger Hu, K. A., Mevi, A. A., Hedderson, M. M., Shan, J., Quesenberry, C. P., \& Ferrara, A. (2014). The Multigroup Ethnic Identity Measure-Revised: Measurement invariance across racial and ethnic groups. Journal of Counseling Psychology, 61(1), 154-161. https://doi.org/:10.1037/a0034749

Bryant, F. B., \& Smith, B. D. (2001). Refining the architecture of aggression: A measurement model for the Buss-Perry Aggression 
Questionnaire. Journal of Research in Personality, 35, 138-167. https://doi.org/:10.1006/jrpe.2000.2302

Buss, A. H. (1961). The psychology of aggression. John Wiley \& Sons Inc. https://doi.org/:10.1037/11160-000

Buss, A. H., \& Durkee, A. (1957). An inventory for assessing different kinds of hostility. Journal of Consulting Psychology, 21(4), 343349. https://doi.org/:10.1037/h0046900

Buss, A. H., \& Perry, M. (1992). The Aggression Questionnaire. Journal of Personality and Social Psychology, 63, 452-459. https://doi.org/:10.1037//0022-3514.63.3.452

Byrne, B. M. (2001). Structural equation modeling with AMOS: Basic concepts, applications and programming. Lawrence Erlbaum.

Castrillón, D., Ortiz, P., \& Vieco, F. (2004). Cualidades paramétricas del Cuestionario de Agresión (AQ) de Buss y Perry en estudiantes universitarios de la ciudad de Medellín (Colombia). Revista Facultad Nacional de Salud Pública, 22(2), 49-61.

Cheung, G. W., \& Rensvold, R. B. (2002). Evaluating goodness-of-fit indexes for testing measurement invariance. Structural Equation Modeling, 9, 233-255. https://doi.org/:10.1207/S15328007SEM0902_5

Coenders, G., Batista-Foguet, J. M., \& Saris, W. (2005). Temas avanzados en modelos de ecuaciones estructurales. Madrid: $\mathrm{La}$ Muralla/Hespérides.

Contini, N. (2015). Agresividad y habilidades sociales en la adolescencia: Una aproximación conceptual. Psicodebate. Psicología, Cultura y Sociedad, 15(2), 31-54.

https://doi.org/:10.18682/pd.v15i2.533

Cruz, R. (2013). Propiedades psicométricas del cuestionario de agresión en dos muestras diferentes de Hidalgo México. European Scientific Journal. 9. 1857-7881.

Featherstone, J. D., \& Zhang, J. (2020). Feeling angry: The effects of vaccine misinformation and refutational messages on negative emotions and vaccination attitude. Journal of Health Communication, 25(9), 692-702. https://doi.org/:10.1080/10810730.2020.1838 671
Fernández-Abascal, E. G., \& Martín Díaz, M. D. (1994). Ira y hostilidad en los trastornos coronarios. Anales de Psicología, 10(2), 177188.

Fossati, A., Maffei, C., Acquarini, E., \& Di Ceglie, A. (2003). Multigroup confirmatory componente and factor anlyses of the Italian version of the Agression Questionnaire. European Journal of Psychological Assessment, 19(1), 54-65. https://doi.org/:10.1027//1015-5759.19.1.54

Gallardo-Pujol, D., Penelo, E., Sit, C., JornetGibert, M. Suso, C., \& Buades-Rotger, M. (2019). The meaning of aggression varies across culture: Testing the measurement invariance of the refined Aggression Questionnaire in samples from Spain, the United States, and Hong Kong. Journal of Personality Assessment 101(5), 515-520. https://doi.org/:10.1080/00223891.2019.1565 572

García-León, A., Reyes, G. A., Vila, J., Pérez, N., Robles, H., \& Ramos, M. M. (2002). The aggression questionnaire: A validation study in student samples. The Spanish Journal of Psychology, 5, 45-53.

https://doi.org/:10.1017/s1138741600005825

George, D., \& Mallery, M. (2010). Using SPSS for Windows step by step: A simple guide and reference. Allyn \& Bacon.

Harrington, N. (2005). The Frustration Discomfort Scale: Development and psychometric properties. Clinical Psychology and Psychotherapy, 12, 374-387. https://doi.org/:10.1002/cpp.465

Hu, L., \& Bentler, P. M. (1999). Cutoff criteria for fit indexes in covariance structure analysis: conventional criteria versus new alternatives. Structural Equation Modeling, 6, 1-55. https://doi.org/:10.1080/10705519909540118

Iacobucci, D. (2010). Structural equations modeling: Fit indices, sample size, and advanced topics. Journal of Consumer Psychology, 20, 90-98.

https://doi.org/:10.1016/j.jcps.2009.09.003

Jiménez-García, P., Contreras, L., Pérez, B., Cova, F., \& Cano-Lozano, M. C. (2020). Adaptación y propiedades psicométricas del Cuestionario de Violencia Filio-Parental (CVIFIP) en jóvenes Chilenos. Revista 
Iberoamericana de Diagnóstico y Evaluación - e Avaliação Psicológica, 56(3), 33-46. https://doi.org/:10.21865/RIDEP56.3.03

Jiménez Gómez, F., Sánchez Crespo, G., Merino Barragán, V., \& Ampudia Rueda, A. (2010). Escala de valoración de la conducta violenta y riesgo de reincidencia (EVCV-RR): Primeros resultados. Revista Iberoamericana de Diagnóstico y Evaluación - e Avaliação Psicológica, 2(30), 87-104. Recuperado de: https://www.redalyc.org/articulo.oa?id=45964 5442006

López, M. C., Sánchez, A., Rodríguez, L. T., \& Fernández, M. P. (2009). Propiedades psicométricas del cuestionario AQ aplicado a población adolescente. EduPsykhé, 8(1), 7994.

López-Cepero Borrego, J., Rodríguez-Franco, L., \& Rodríguez-Díaz, F. J. (2015). Evaluación de la violencia de pareja. Una revisión de instrumentos de evaluación conductual. Revista Iberoamericana de Diagnóstico y Evaluación - e Avaliação Psicológica, 2(40), 37-50.

Recuperado en:

https://www.redalyc.org/articulo.oa?id=45964 5432005

Lovas, L., \& Trenkova, S. (1996). Aggression and perception of an incident. Studia Psychologia, 38, 265-270.

Matalinares Calvet, M., Yaringaño L., J., Uceda E., J., Fernández A., E., Huari T., Y., \& Campos G., A. (2012). Estudio psicométrico de la versión española del cuestionario de agresión de Buss y Perry. Revista De Investigación En Psicología, 15(1), 147-161. https://doi.org/:10.15381/rinvp.v15i1.3674

Maxwell, J. P. (2007). Development and preliminary validation of a Chinese version of the Buss-Perry Aggression Questionnaire in a population of Hong Kong Chinese. Journal of Personality Assessment, 88, 284-294. https://doi.org/:10.1080/00223890701317004

Medrano, L. A., \& Muñoz-Navarro, R. (2017). Aproximación conceptual y práctica a los modelos de ecuaciones estructurales. Revista Digital de Investigación en Docencia Universitaria, 11, 219-239.

https://doi.org/:10.19083/ridu.11.486
Medrano, L., \& Moretti, L. (2015). Prácticas basadas en la evidencia: Ciencia y profesión en el campo de la salud. Editorial Brujas.

Medrano, L., Franco, P., \& Mustaca, A. (2018). Adaptación argentina de la "Escala de Intolerancia a la Frustración. Behavioral Psychology, 26(2), 303-321.

Meesters, C. M., Muris, P., Bosma, H., Schouten, E., \& Beuving, S. (1996). Psychometric evaluation of the Dutch version of the Aggression Questionnaire. Behaviour Research and Therapy, 34, 839-843. https://doi.org/:10.1016/0005-7967(96)00065-4

Nakano, K. (2001). Psychometric evaluation of the Japanese adaptation of the Aggression Questionnaire. Behaviour Research and Therapy, 39(7), 853-858. https://doi.org/:10.1016/S00057967(00)00057-7

Pechorro, P., Barroso, R., Poiares, C., Oliveira, J., \& Torrealday, O. (2015). Validation of the Buss-Perry Aggression Questionnaire-Short Form among Portuguese juvenile delinquents. International Journal of Law and Psychiatry, $44,75-80$. https://doi.org/:10.1016/j.ijlp.2015.08.033.

Pfister, R., \& Masse, P. (2001). Le Questionnaire d'Agressivité: Validation et équivalence transculturelle. IXème Congrès International des Chercheurs en Activités Physiques et Sportives, Valencia, Francia.

Porras, S., Salamero, M., \& Sender, R. (20012002). Adaptación española del Buss-Perry Aggression Questionnaire [Spanish adaptation of the Buss-Perry Aggression Questionnaire]. Cuadernos de Medicina Psicosomática y Psiquiatría de Enlace, 60/61, 7-12.

Ramirez, J. M., Andreu, J. M., \& Fujihara, T. (2001). Cultural and sex diferences in agression: A comparison between Japanese and Spanish students using two different inventories. Aggressive Behavior, 27(4), 313322. https://doi.org/:10.1002/ab.1014

Redondo, N., Peña, M. E., Graña, J. L., \& Andreu, J. M. (2017). Psychometric properties of the Aggression Questionnaire: A replication in a sample of partner-assaultive men in psychological treatment. Psicothema, 29(4), 584-589.

https://doi.org/:10.7334/psicothema2016.390. 
Reyna, C., Lello, M. G., Sanchez, A. y Brussino, S. (2011). The Buss-Perry Aggression Questionnaire: construct validity and gender invariance among Argentinean adolescents. International Journal of Psychological Research, 4, 30-37. Recuperado de https://www.redalyc.org/articulo.oa?id=29902 3516004

Ruchkin, V. V. y Eisemann, M. (2000). Aggression and psychological problems in juvenile male delinquents versus controls in Russia: alternate ways of "letting off steam"?. Aggression and Violent Behavior, 5, 217-225. https://doi.org/:10.1016/S13591789(98)00037-8

Santisteban, C., Alvarado, J. M. y Recio, P. (2007). Evaluation of a Spanish version of the Buss and Perry Aggression Questionnaire: some personal and situational factors related to the aggression scores of young subjects. Personality and Individual Differences, 42, 1453-1462.

https://doi.org/:10.1016/j.paid.2006.10.019

Sierra, J. C. y Gutiérrez, R. (2007). Validación de la versión española del Cuestionario de agresión de Buss-Perry en estudiantes universitarios salvadoreños. Psicología y Salud, 17, 103-113.

Sommantico, M., Osorio, M., Parrello, S., De Rosa, B. y Donizzetti, A. R. (2008). Validación de la versión italiana del Cuestionario de agresión (AQ) en el sur de Italia. Revista Electrónica de Psicología Iztacala, 1, 28-45.

Tremblay, P. F. y Ewart, L. A. (2005). The Buss and Perry Aggression Questionnaire and its relations to values, the Big Five, provoking hypothetical situations, alcohol consumption patterns and alcohol expectancies. Personality and Individual Differences, 38, 337-346. https://doi.org/:10.1016/j.paid.2004.04.012

Valdivia-Peralta, M., Fonseca-Pedrero, E., González-Bravo, L., \& Lemos-Giráldez, S. (2014). Psychometric properties of the AQ Aggression scale in Chilean students. Psicothema, 26, 39-46.

https://doi.org/:10.7334/psicothema2013.84

Vigil-Colet, A., Lorenzo-Seva, U., CodorniuRaga, M. J., \& Morales, F. (2005). Factor structure of the Buss-Perry aggression questionnaire in different samples and languages. Aggressive Behavior, 31(6), 601608. https://doi.org/:10.1002/ab.20097

Vinagre-González, A. M., Aparicio-García, M. E., \& Alvarado-Izquierdo, J. M. (2020). IVISEM, una medida de la violencia social encubierta hacia las mujeres. Revista Iberoamericana de Diagnóstico y Evaluación - e Avaliação Psicológica, 57(4), 149-161. https://doi.org/: 10.21586/RIDEP57.4.11

Vitoratou, S., Ntzoufras, I., Smyrnis, N., \& Stefanis, N. (2009). Factorial composition of the Aggression Questionnaire: A multi-sample study in Greek adults. Psychiatry research. 168. 32-9.

https://doi.org/:10.1016/j.psychres.2008.01.016

Von Collani, G., \& Werner, R. (2005). Selfrelated and motivational constructs as determinants of aggression. An analysis and validation of a German version of the BussPerry Aggression Questionnaire. Personality and Individual Differences, 38, 1631-1643. https://doi.org/:10.1016/j.paid.2004.09.027

Williams, T. Y., Boyd, J. C., Cascardi, M. A., \& Poythress, N. (1996). Factor structure and convergent validity of the aggression questionnaire in an offender population. Psychological Assessment, 8(4), 398-403 https://doi.org/: 10.1037//1040-3590.8.4.398.

World Health Organization (2003) Informe Mundial sobre la Violencia y la Salud [World Report on Violence and Health]. Organización Panamericana de la Salud. 